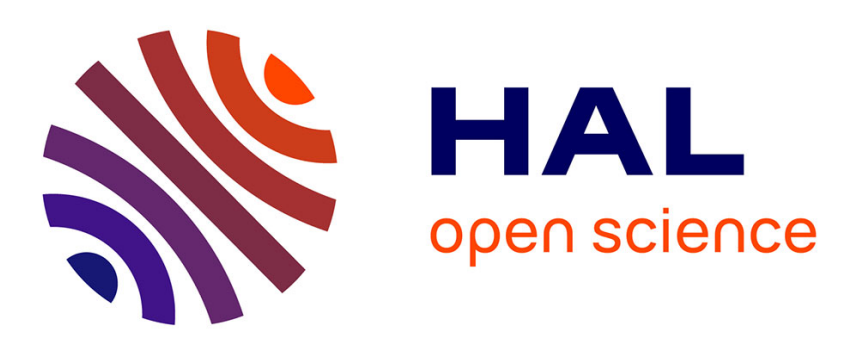

\title{
Thermal imaging of the structural damping induced by an acoustic black hole
}

Thomas Durand-Texte, A. Pelat, Guillaume Penelet, F. Gautier, Mathieu Secail-Geraud

\section{- To cite this version:}

Thomas Durand-Texte, A. Pelat, Guillaume Penelet, F. Gautier, Mathieu Secail-Geraud. Thermal imaging of the structural damping induced by an acoustic black hole. Journal of Applied Physics, 2020, 127 (2), pp.025102. 10.1063/1.5128089 . hal-02453078

HAL Id: hal-02453078

https://hal-univ-lemans.archives-ouvertes.fr/hal-02453078

Submitted on 6 Apr 2020

HAL is a multi-disciplinary open access archive for the deposit and dissemination of scientific research documents, whether they are published or not. The documents may come from teaching and research institutions in France or abroad, or from public or private research centers.
L'archive ouverte pluridisciplinaire HAL, est destinée au dépôt et à la diffusion de documents scientifiques de niveau recherche, publiés ou non, émanant des établissements d'enseignement et de recherche français ou étrangers, des laboratoires publics ou privés. 


\title{
Thermal imaging of the structural damping induced by an acoustic black hole
}

\author{
T. Durand-Texte, ${ }^{1}$ A. Pelat, ${ }^{1}$ G. Penelet, ${ }^{1}$ F. Gautier, ${ }^{1}$ and M. Sécail-Géraud ${ }^{1}$ \\ LAUM UMR CNRS 6613, avenue Olivier Messiaen, F-72085 Le Mans cedex 9, France
}

An Acoustic Black Hole (ABH) is a passive and efficient way to control the flexural vibrations of beams or plates. In its classical form, an $\mathrm{ABH}$ consists in a local reduction of the thickness of a structure according to a power law profile, associated to a thin visco-elastic coating placed in the thinnest region. A focalisation and a wave trap effect occur, leading to a localised energy dissipation which induces a local temperature increase. The objective of this paper is twofold. Firstly, the goal is to develop an adequate experimental methodology enabling to map accurately the small temperature variations induced by the local dissipation mechanism. Secondly, from the thermal standpoint, the goal is to provide experimental evidence of a local temperature increase associated to a damping effect in the case of an $\mathrm{ABH}$ beam. This paper thus describes a new kind of experimental methodology able to provide original data, bringing some new insight in the $\mathrm{ABH}$ physical understanding and the analysis of structural damping.

\section{INTRODUCTION}

Using the Acoustic Black Hole (ABH) effect is a way to passively control vibrations without adding mass. The potential use of this technique mainly targets niche applications in which the weight of the devices used for vibration control is a constraint. This is typically the case, for instance, for the automotive industry, where the major challenge is to guarantee the reliability of the structure and a low level of interior noise with a limited global mass for the vehicle.

For a simple vibrating structure like a beam, an $\mathrm{ABH}$ termination can be achieved by locally reducing the thickness of the beam according to a power law profile. In the ideal case, originally introduced by Mirono $\sqrt{1}$, the thickness should decreases down to zero. As a consequence, for an incident wave incoming to the edge, the wave speed along this profile decreases down to zero and the time of flight tends towards infinity. In parallel, the amplitude of the wave increases up to infinity, due to energy flow conservation. Thus, this ideal case corresponds to a singularity, for which the mechanical reflection coefficient is equal to zero, without any dissipation. In practice, the ideal $\mathrm{ABH}$ cannot be perfectly manufactured: as the end of the profile is necessarily truncated, the residual thickness of the beam at its extremity cannot be equal to zero. This defect induces a significant wave reflection which can be compensated by gluing a thin visco-elastic layer along the tapered region ${ }^{2}$. Such an ABH presents a low reflection coefficient.

In the literature, various models have been developed ${ }^{\sqrt{3}+6}$ to give some understanding of $\mathrm{ABH}$ features which can be identified experimentally $y^{7}$, for instance using the impedance matrix method for plane waves ${ }^{\sqrt{3}}$, multimoda ${ }^{4}$ models, wavelet ${ }^{5}$ or the hypergeometric approach 6 , among others. It is notably shown that the typical arched shape of the reflection coefficient curve is due to resonances of the complex modes trapped in the $\mathrm{ABH}$ ending 89 , thoroughly studied over the past years 4 10 13 . Indeed, all the published studies show that the $\mathrm{ABH}$ effect, either in 1D or 2D structures, leads to efficient energy dissipation 14 . Various designs of the ABH devices have been proposed by optimising the thickness profile ${ }^{15} \mid 19$, but the damping profile itself is rarely fully optimised.

Recent studies have shown that tuning the damping in a profiled beam termination results in a critical coupling of the complex modes trapped within the ABH taper ${ }^{13}$ : the value of the reflection coefficient can indeed achieve zero at a given frequency corresponding to the resonance of a local mode. Consequently, controlling the amount of damping is a key point to achieve critical coupling, and this can be done for instance with active methods using polymers whose damping properties are controlled by adjusting their temperature20.

All in all, controlling the damping profile is one of the promising lines aiming at improving the design of $\mathrm{ABH}$ endings. This prospect also relies on the use of advanced measurement techniques to characterise experimentally the dissipation of energy. Although inverse techniques result in such a characterisation for relatively simple academic cases ${ }^{21}$, mechanical damping is usually complex to model because of the multiple mechanisms involved: visco-elasticity, thermoelasticity, viscous coupling with the surrounding air, micro friction forces at the interfaces... All these mechanisms lead to energy conversion into heat, and thus in fine to a local temperature increase ${ }^{22}$, which, in turn, may be considered as a global indicator allowing to identify the regions where dissipation occurs, without any distinction regarding their physical origin. This is typically the main interest in thermal measurements applied to vibrations ${ }^{23 \cdot 27}$, aiming at optimising the mechanical parameters and adjusting them for specific needs, for instance for cardboards $\sqrt{28}$ or rubbers 29 .

The work presented in this paper aims at developing an experimental methodology in order to characterise the spatial distribution of mechanical losses in vibrating thin structures, by means of thermal map measurements. The development of an experimental technique enabling to measure accurately the temperature distribution on a vibrating structure is presented; this technique is then applied to the thermal imaging of an $\mathrm{ABH}$ beam termination. Such thermal imaging of an $\mathrm{ABH}$ is, to the authors' knowledge, the main novelty provided by the paper. The major difficulty is indeed here to measure small temperature variations (therefore with a low signal to noise ratio) resulting from the local dissipation of mechanical energy. In order to address this objective, an infra-red camera is used and an adequate signal and image processing has been implemented. The paper is organised as follows. Firstly, section II details the experimental set-up (Sec. IIA) and the 
mechanical characterisation with usual methods (Sec. IIB). Secondly, the experimental protocol and the image processing of raw data extracted from the infrared camera are presented in section III] Thirdly, the temperature variations generated by mechanical damping are experimentally described in section IV]A. Finally, the transient variation of the thermal profile of the $\mathrm{ABH}$ is studied in section IV $\mathrm{B}$.

\section{EXPERIMENTAL SET-UP AND MECHANICAL CHARACTERIZATION}

\section{A. Experimental set-up}

A sketch of the experimental set-up is presented in Fig. 1. (a). The device consists of an aluminum beam terminated with the $\mathrm{ABH}$ at the bottom end. Apart from the black hole termination, the beam is $605 \mathrm{~mm}$ long, $20 \mathrm{~mm}$ wide, and $5 \mathrm{~mm}$ thick. Flexural waves can be generated along the beam by means of a dynamic shaker, whose location is close to the top end at position $x=-500 \mathrm{~mm}$ (see Fig. 1](a)). In order to decouple the system from external vibration noise, both the beam and the shaker are hung to two different gallows screwed to a marble plane set on dampers.

The $\mathrm{ABH}$ is located at position $x=0$, where the thickness of the beam decreases slightly down to $290 \mu \mathrm{m}$ over about $155 \mathrm{~mm}$ and is extended by a $25 \mathrm{~mm}$ long plateau of constant thickness. The plateau leads to a significant enhancement of the $\mathrm{ABH}$ effect, and an appreciable shift of the frequency band, for which the $\mathrm{ABH}$ is active, toward low frequencies 30 . Considering the $\vec{x}$ axis defined in Fig. 1. (a), the thickness variations are given by:

$$
h(x)= \begin{cases}h_{0}\left(1-x / x_{0}\right)^{2}, & \text { if } x_{P} \geq x \geq 0 \\ \approx h_{P}, & \text { if } x \geq x_{P}\end{cases}
$$

with $h_{0}(50 \mathrm{~mm})$ the uniform thickness of the beam, $x_{0}$ $(204 \mathrm{~mm})$ the position at which $h(x)=0, x_{P}(155 \mathrm{~mm})$ the position at which the thickness remains constant and $h_{P}$ $(290 \mu \mathrm{m})$ the thickness of the plateau. In order to provide some evidence of the local temperature increase due to the dissipation of mechanical power, the naked (without added layer) and coated (with added layer) ABH configurations are compared (see Fig. 1 b). The visco-elastic layer is a double-sided tape, made of a cotton fabric coated with a rubber adhesive and a silicon liner (supplier https://www.foussier.fr. product ref: 438347). The estimated thickness of the layer is about $230 \mu \mathrm{m}$. The resulting dissipation mechanism is characterised through thermal measurements using an infrared camera (FLIR X6580c), combined to vibration field measurements carried out with a Scanning Laser Doppler Vibrometer (SLDV, Polytec PSV500Xtra). Both devices are placed in front of the beam, at a distance of $1170 \mathrm{~mm}$ and $760 \mathrm{~mm}$ respectively ( $c f$. Fig. 1 (a), where the SLDV is placed behind the beam for the sake of visibility). A shaker excites the beam $500 \mathrm{~mm}$ far from the $\mathrm{ABH}$ and an impedance sensor (PCB288d01) is used to measure the mechanical impedance at the driving point. Finally, the front (measured) side of the

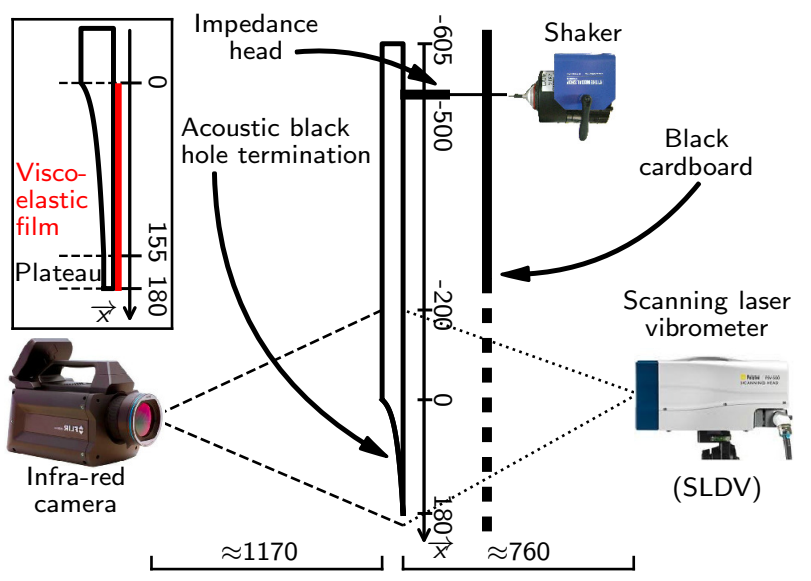

(a)

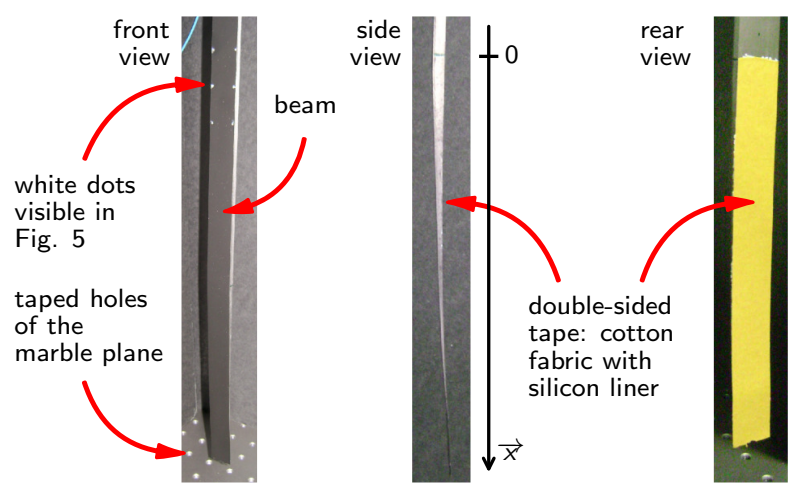

(b)

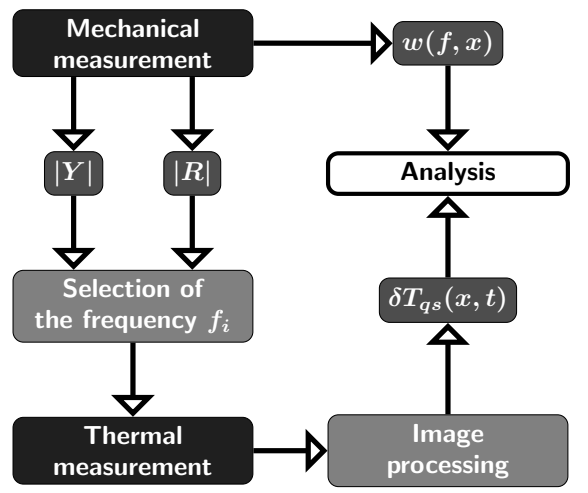

(c)

FIG. 1. (a) Sketch of the experimental set-up, with dimensions expressed in $\mathrm{mm}(\mathrm{ABH}$ begins at $x=0)$; (b) pictures of the $\mathrm{ABH}$ (covered with visco-elastic material), front, side and rear views; the front (measured) side is painted black ; the profile of the $\mathrm{ABH}$ is visible on the side view; the rear view shows the $\mathrm{ABH}$ with the viscoelastic film; (c) block diagram of the proposed method.

beam is painted black (cf. Fig.11.(b)) and the experimental setup is fully cloaked with black cardboards during thermal measurements in order to maximise the thermal emissivity and to reduce the noise from the environment in the measured thermograms.

A block diagram of the proposed method is presented in Fig. 1(c). The mechanical data obtained with the impedance 
head and SLDV, the admittance $Y$ and the reflection coefficient $R$, are used to select frequencies for infra-red imaging recordings. The obtained thermograms are then processed to calculate the quasi-static temperature variations $\delta T_{q s}(x, t)$, which are finally analysed, along with the vibrational displacements $w(x, t)$, in order to gain insight into the physical behaviour of the $\mathrm{ABH}$ and structural damping.

\section{B. Mechanical characterisation of the $A B H$}

From a mechanical standpoint, two indicators are used to assess the effect of damping in the coated case:

1. the co-localised admittance $Y=V / F$ of the sample beam (where $V$ and $F$ are the velocity and the force at the driving point);

2. the mechanical reflection coefficient $\mathrm{R}$ of the $\mathrm{ABH}$ termination that can be measured by following the method described in Ref. 7.

Far from the shaker and from the $\mathrm{ABH}$ termination, (i.e. in the far-field condition), the spatial distribution of the displacement along the vibrating beam can be written as the sum of two counter-propagative waves, whose unknown complex amplitudes can be determined by means of a least mean square method from the SLDV measurements performed on a grid of points.

The modulus of the admittance $Y$ shown in Fig. 2 (a) displays a succession of peaks and drops, whose frequency is determined by the beam dimensions, in particular the total length. In the naked case, these peaks/drops are sharp due to the low loss factor of the constitutive material of the beam, and to the total reflection at the extremity $(|R| \approx 1$ in the naked case in Fig. 2(b)). In the coated case, $|Y|$ is significantly smoothed due to the ABH performances. Indeed, the modulus of $R$ (Fig.2(b)) decreases when the frequency increases, and displays typical arches for which each drop corresponds to the resonance of a mode localised in the ABH profile 813 . These trapped modes are independent from the length of the beam. Hence, the frequencies corresponding to the drops of $|R|$ are not directly linked to those of the peaks/drops of IYI.

In addition to the frequency responses of the structure presented in Fig. 22 measurements have also been performed to characterize the spatial distribution of the vibrational field. Fig. 3. (a) shows a typical map of the out-of-plane forced displacement (normalised to its maximum value) in the case of the coated $\mathrm{ABH}$, at $1,000 \mathrm{~Hz}$. The field clearly shows the increase along the $\mathrm{x}$-axis of the amplitude of vibration, as well as a reduction of the wavelength, which are both induced by the $\mathrm{ABH}$ ending. In the frequency range of interest, it must be noticed that the vibrational field only depends on the position along the x-axis, and not on the y-axis. Fig. 3(b) represents the flexural field in a frequency $v s$ space plane in which a horizontal line corresponds to the longitudinal profile of the field at a given frequency, obtained by averaging the field as measured in Fig. 3. (a) over the width of the beam. Fig. 4 gives examples of such profiles with or without a damping layer, at

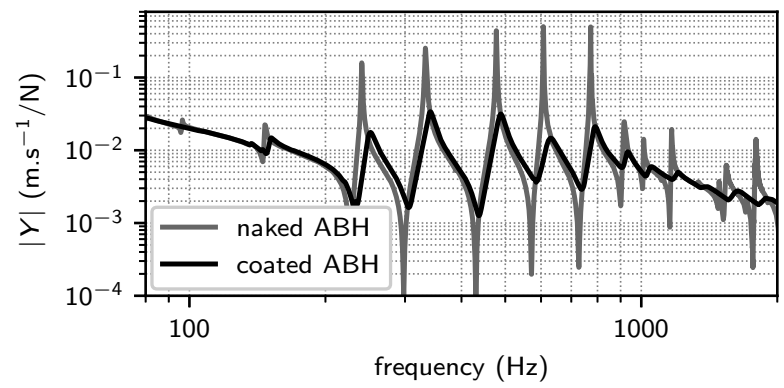

(a)

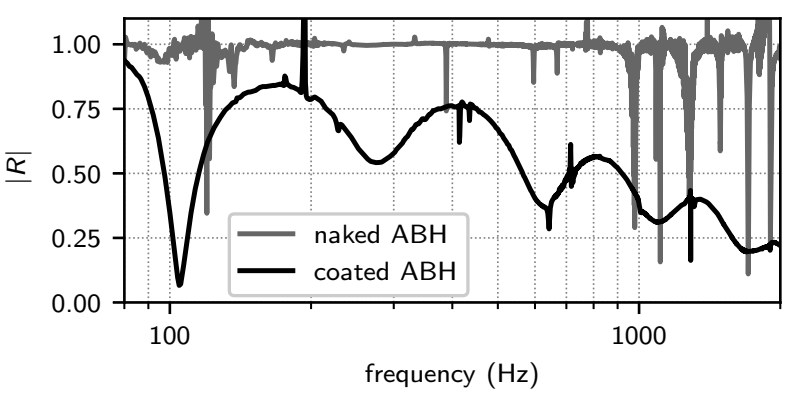

(b)

FIG. 2. (a) Magnitude of the mobility of the excitation point ( $x=$ $-500 \mathrm{~mm}$ ); (b) modulus of the reflection coefficient of the (black) coated and (gray) naked ABH calculated at $x=0$. A $50 \mathrm{~mm}$ wide grid of $26 \times 6$ measurement points located in the region $x<-125 \mathrm{~mm}$ is used in order to verify the far-field conditions.

two selected frequencies. It must be noted that the excitation frequency is not set exactly at the same value for the coated or for the naked cases: this is because the frequency is chosen so as to correspond to a local maximum of the admittance, which itself is influenced by the presence of the viscoelastic layer. The results clearly show localised vibrations in the $\mathrm{ABH}$ and a huge amplitude reduction in the coated $\mathrm{ABH}$ case, compared with the typical amplitudes of the naked $\mathrm{ABH}$ case.

\section{THERMAL METROLOGY}

\section{A. Experimental set-up for IR-thermography}

This part is devoted to metrological aspects and to the processing of raw data applied to the thermal measurements using the infra-red camera. The data obtained with the infrared camera correspond to the surface temperature of the aluminum beam, which itself can be decomposed into two components as:

$$
T(x, t)=T_{0}(x)+\delta T_{q s}(x, t) .
$$

In Eq. 22), the temperature $T_{0}(x)$ corresponds to a reference temperature, namely that of the beam at rest. This reference temperature is actually very close to the room temperature $T_{\infty}$, but it may be slightly influenced by parasitic sources of heating. The quasi-static temperature, $\delta T_{q s}(x, t)$, corresponds to the measurand of interest in this study. It refers to the space- 


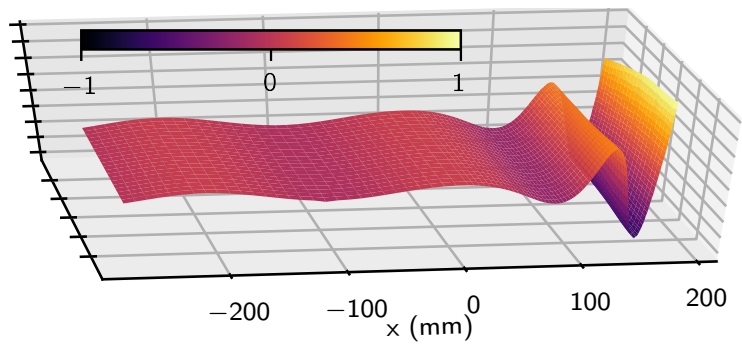

(a)

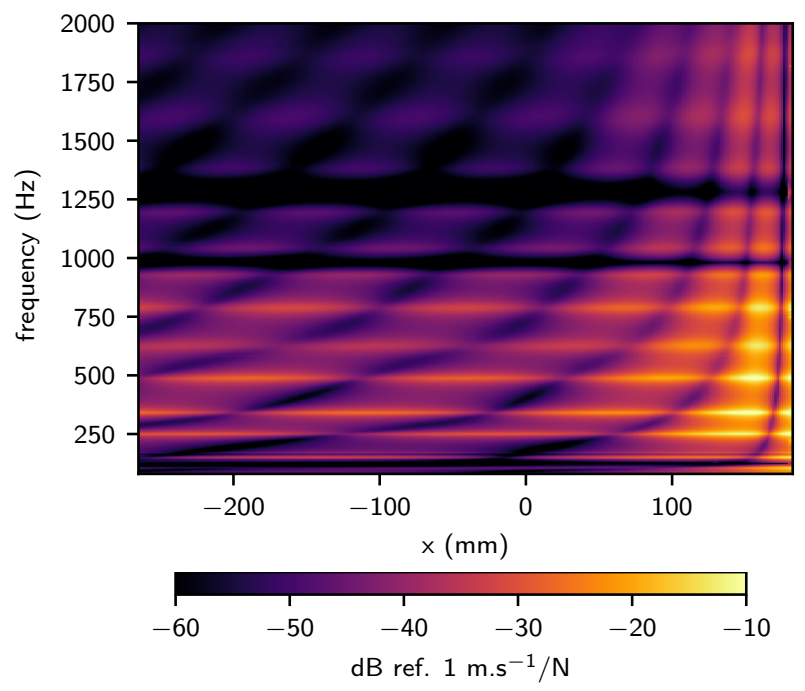

(b)

FIG. 3. (a) Snapshot of the measured velocity of the coated ABH at $1,000 \mathrm{~Hz}$; the $\mathrm{ABH}$ begins at $x=0$ and the amplitude is normalised to its maximum value; (b) absolute value of the frequency response functions $Y(\omega, x)=V(\omega, x) / F(\omega)$ where $V(\omega, x)$ is the velocity spectrum, at point $\mathrm{x}$ belonging to the bottom end of the coated $\mathrm{ABH}$, and where $F(\omega)$ is the applied force spectrum at the driving point.

and time-dependent component of the temperature distribution induced by the dissipation of mechanical power when flexural waves are generated along the beam. It is worth pointing out that in Eq. (2), we deliberately discarded the fact that there should be an additional term, corresponding to an oscillating component of the temperature variations. The flexural waves in the beam should indeed also lead to some fluctuations of temperature resulting from the thermo-elastic effect caused by a dynamic change in elastic strain. However, this oscillating component of temperature fluctuations, whose time-average over one cycle equals zero, is not considered in this study. Its order of magnitude is indeed assumed to be small against the slow variations $\delta T_{q s}$ of the temperature caused by damping, and the exposure time used in this study for temperature measurements (about $3 \mathrm{~ms}$ ) is comparable to the period of one cycle (typically from 1 up to $10 \mathrm{~ms}$ ). It is worth noting that, in Eq. (2), the temperature is assumed to depend only on the axial component, $x$ : this statement is actually confirmed by the observations in the experiments carried out for the present study.

The camera used is a FLIR X6580sc, with a sensor matrix
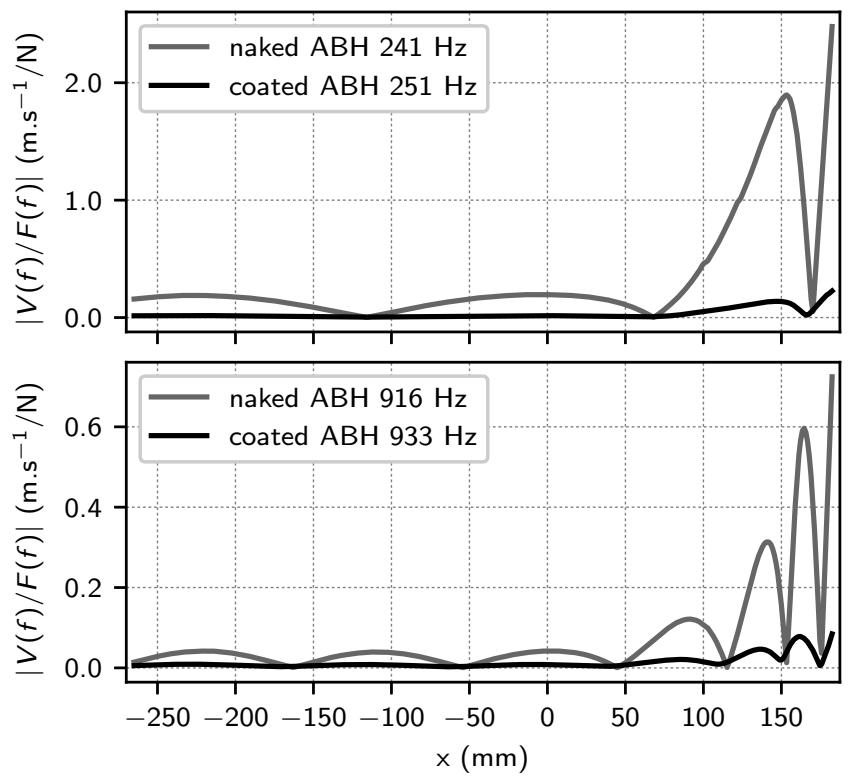

FIG. 4. Vibrational profiles of the bottom part of the beam for two modes, $241 \mathrm{~Hz}$ and $916 \mathrm{~Hz}$ for the naked $\mathrm{ABH}$ and $251 \mathrm{~Hz}$ and $933 \mathrm{~Hz}$ for the coated $\mathrm{ABH}$ ).

of $512 \times 640$ pixels. Its thermal sensitivity is about $20 \mathrm{mK}$, with an absolute and a relative uncertainty equal to $\pm 1^{\circ} \mathrm{C}$ and $\pm 1 \%$ respectively. In order to minimise the noise potentially visible in the thermograms, the whole set-up is cloaked with black cardboards and the beam is painted black to maximise the thermal emissivity. Note that the infra-red radiation emitted by the camera itself is reflected on the beam, even if the beam is painted black, and this radiation is clearly visible on the thermograms, except if the camera is voluntarily not placed in front of the beam but slightly aside.

The non-uniformity of the thermal maps is corrected before every measurement phase: the software driving the infra-red camera allows correcting the non-uniformity from images of a scene of uniform temperature (by using a black cardboard placed in front of the objective of the camera for example). An example of a thermogram of the beam, with the correction of the non-uniformity done beforehand, is presented in Fig. 5 . As shown in Fig. 1.(b), white dots are painted on the beam, and are also visible in the thermogram in Fig. 5. These dots give reference positions and enable retrieving the system of coordinates used both for SLDV and IR thermometry. On the thermograms presented (Fig. 5 and 7.(a)), the black hole side $(x>0)$ is on the right.

When observing the evolution of the quasi-static temperature profile of the beam at rest, measured at several times $\left(\delta T_{q s}\left(x, t_{i}\right)\right)$, two types of variations are noticeable: small variations on each image (around a few $\mathrm{mK}$ ) and jumps (around $90 \mathrm{mK}$ ). After several observations, the former look uniform, whereas the latter display spacial coherence. The small variations are due to the temperature drift of the body of the camera and the jumps are due to the correction of this drift, done automatically by the integrated software. Note that the values of these variations remain in the range of the uncertainty values 


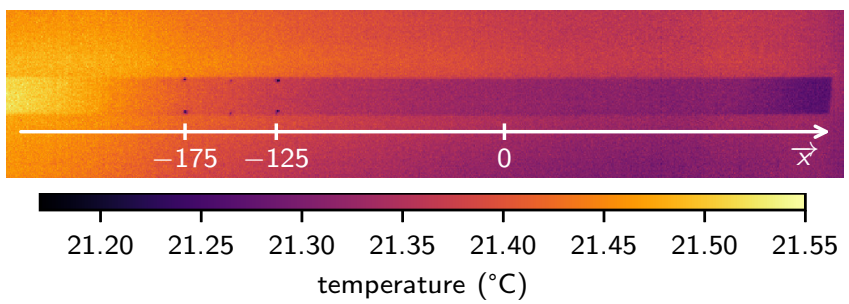

FIG. 5. Example of thermogram of the beam at rest, averaged in time over 30 thermograms $\left(15 \mathrm{sec}\right.$.), used to measure $T_{0}(x)$.

given by the manufacturer. However, these variations can be corrected by processing the signal. As the objectives of this experiment require to measure variations of around a few tens of $\mathrm{mK}$, signal and image processing is thus used to lessen the uncertainty values and improve the accuracy of the measurement.

\section{B. Image processing}

As mentioned previously, the thermograms measured present small uniform variations and the software integrated in the camera corrects the drift by taking into account the temperature of the body of the camera, which leads to jumps in the measured signals. These jumps are not uniform; yet they seem spatially coherent. As the variable of main interest is the quasi-static one, $\delta T_{q s}(x, t)$, and is about a few tens of $\mathrm{mK}$, these jumps must be corrected. As the drift correction cannot be switched off, the protocol used consists firstly in suppressing manually the automatic correction, which simultaneously removes the jumps, and secondly in correcting manually the temperature drift. The different steps of the processing are summarised in Fig. 6

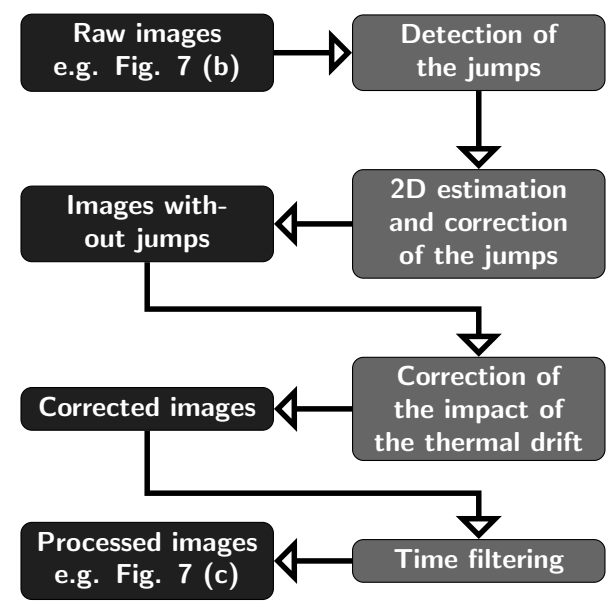

FIG. 6. Synoptic diagram of the image processing applied; (black) type of images and (grey) image processing stages.

Firstly, the temperature variation, spatially averaged in each raw image, is calculated so as to detect the jumps. For each jump detected in time, the variations of $\delta T_{q s}(x, t)$ between two thermograms are supposedly very small compared to the jumps. 2D estimations of the variations due to these jumps are then calculated from the difference between the averaging of $n$ thermograms before and after each jump (here $n=3$ ), by using the least mean square method, with a 3-order polynome. These $2 \mathrm{D}$ estimations are subsequently used to remove the jumps.

As mentioned previously, the signal drift due to the temperature variation of the body of the camera appears, and is thus supposed uniform in all the thermograms. The impact of this thermal drift is finally estimated by using a black cardboard set behind the beam, in the background. It corresponds to the average temperature variation of this black area, whose temperature is assumed constant during each acquisition. This value is then subtracted from the thermograms in order to get the corrected images. The final step consists in filtering the signal of each pixel in time, to smooth the results. To illustrate this protocol, three measured signals of the beam undergoing a local increase in temperature are shown in Fig.77. before and after processing. The frame rate of the camera is set to 2 frames per second (fps). Before the processing stage, noise, jumps and signal drift are visible. After processing, the curves are visibly smoother, the jumps have been removed and the drift is corrected.

\section{THERMO-MECHANICAL CHARACTERISATION OF THE ABH}

\section{A. Evidence of the thermal increase due to the added damping layer}

This section aims at highlighting local heating due to the added damping layer when the beam is excited in a steady state by a wideband noise over the range $[0.2-1] \mathrm{kHz}$. The frame rate of the infra-red camera is set to $2 \mathrm{fps}$, and the exposure time to $3,000 \mu$ s.

The choice of the voltage supplied to the dynamic shaker is a tricky issue in this experiment. Ideally, the same power should be injected for both cases of naked and coated ABH. In practice, this setting is impossible to achieve due to the differences in the order of magnitude of the mechanical admittance of the beam between the two cases, as discussed for Fig. 3. (a). Indeed, applying a power level so that a heating may be measurable in the coated case results in a highly nonlinear vibration regime in the naked case. Conversely, applying a power level that guarantees a linear vibration regime in the naked case results in very low power dissipation within the $\mathrm{ABH}$ and the resulting local increase in temperature is not possible to measure.

The adopted compromise then consists in adjusting the power level so that its value $2\left|\mathfrak{R}\left\{S_{f a} / j \omega\right\}\right|$ ( $S_{f a}$ being the cross-spectrum force-acceleration $\sqrt{31 \mid 32}$ may be of the same range at resonance peaks in both naked and coated cases (see Fig. 8). Therefore, due to large differences in damping between the two cases, the total injected energy that corresponds to the frequency integration of these signals is significantly different: the values obtained are $21.6 \mathrm{~mW}$ and 


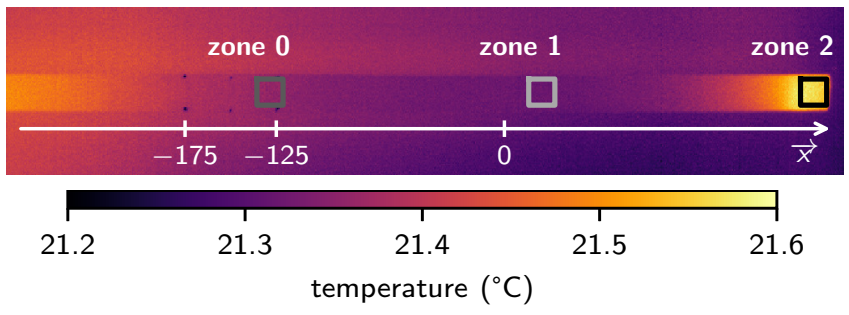

(a)

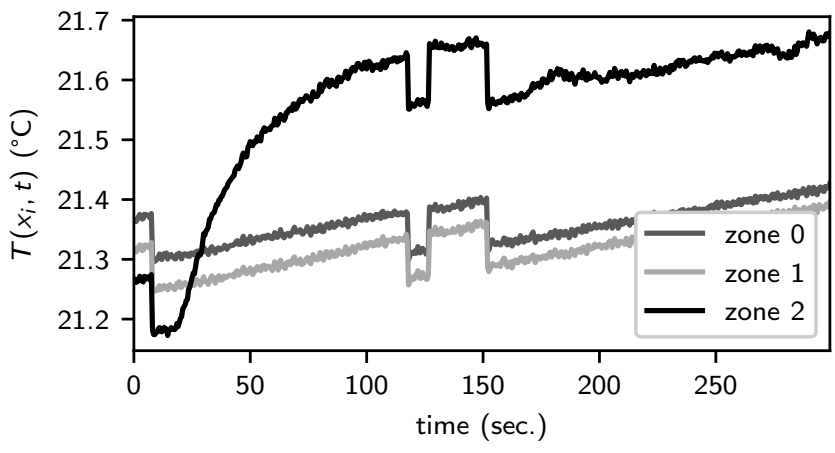

(b)

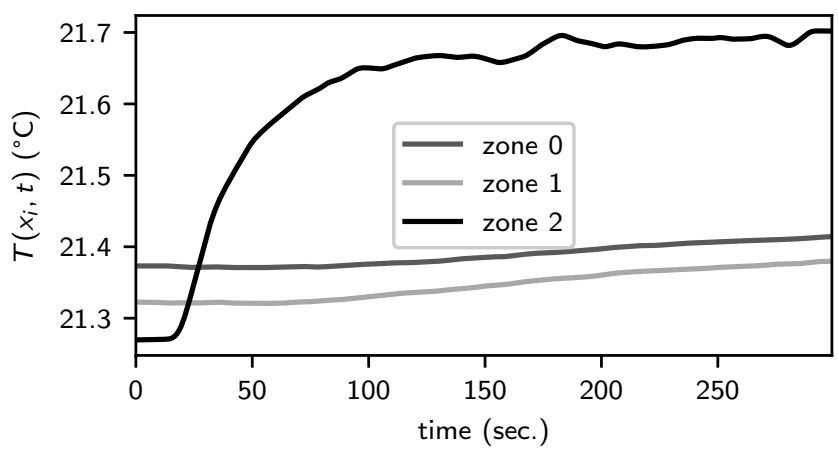

(c)

FIG. 7. (a) Time averaged thermogram (over $300 \mathrm{sec}$.) and measurement areas: (black) zone 0, (light gray) zone 1, (dark gray) zone 2; the variations of the average temperature, plotted for the three areas, are estimated (b) from raw images, and (c) from processed images.

$245.5 \mathrm{~mW}$ in the naked and coated cases, respectively. These values reflect the differences in the level of energy dissipated by the $\mathrm{ABH}$ in both cases.

Fig. 9 shows the evolution of the quasi-static temperature $\delta T_{q s}(x, t)$ over a long time after having switched on the broadband excitation at $t=20 \mathrm{sec}$. Each vertical column of this plane is obtained by averaging the instantaneous thermogram over the width of the beam.

In the naked ABH (see Fig.9.(a)), no significant heating is measured on the vibrated beam compared to its thermal state at rest. However, slight and abrupt temperature variations of a few tens of $\mathrm{mK}$ with complex time evolution are observed. These variations are probably due to uncontrolled environmental conditions in the set-up, such as slight air streams and room temperature variations or light pollution.

However, for the coated case presented in Fig. 9.(b), a localised heating at the end of the beam, where the vibration

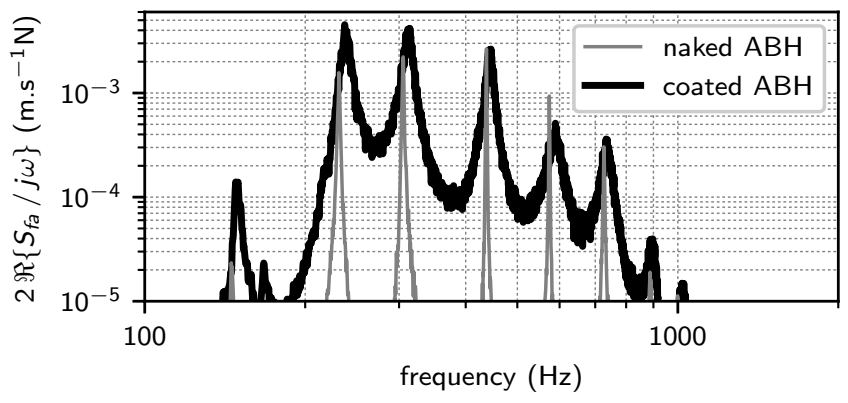

FIG. 8. Measurement of the injected power, $\Pi(\omega)=2 \mathfrak{R} S_{f a} / j \omega, S_{f a}$ beeing the cross-spectrum force - acceleration, for the naked and the coated $\mathrm{ABH}$.

amplitudes and dissipative mechanisms are greater, is clearly measured. After a transient rise of about $30 \mathrm{sec}$. after switching on the source, the temperature profile stabilises and shows a maximum value of about $500 \mathrm{mK}$. In these conditions, the $\mathrm{ABH}$ is active and generates a local increase of the temperature. These experimental data provide the main original result of this paper.

\section{B. Analysis of the transient evolution of the thermal profile}

It is also relevant to analyse the variations the temperature field $\delta T_{q s}$ in both space and time domains, in the case of a harmonic excitation. Such an analysis of the transient establishment of the temperature distribution is provided hereafter. Fig. 10 (a) and Fig. 11 (a) show the evolution of $\delta T_{q s}\left(x, t_{i}\right)$, for $t_{i}$ ranging from $18 \mathrm{sec}$. (when the shaker is switched on) to $50.5 \mathrm{sec}$. (when a steady state is reached) with time steps of $2.5 \mathrm{sec}$., for a sinusoidal excitation at $276 \mathrm{~Hz}$ and $933 \mathrm{~Hz}$. These two frequencies are close to those of vibrational modes of the beam terminated with a coated ABH (see Fig. 4 (a)). The corresponding reflection coefficients are similar and equal to 0.54 and 0.49 , respectively. The frame rate of the infra-red camera is set to $2 \mathrm{fps}$. Moreover, in order to smooth the thermograms, a space Gaussian filter has been applied with the filtfilt algorithm 33 .

Has the dissipated power is usually related to the strain energy, the second spatial derivative of the deformation, $\partial^{2} w(x) / \partial x^{2}$, is estimated from data obtained with SLDV as it is proportional to the strain 34 . The corresponding curves are displayed in Fig. 10 (b) and Fig.11.(b) for both frequencies.

Two conclusions derive from the analysis of these curves:

- for both chosen frequencies, the position where heating is the greatest does not correspond to the position where the vibrational amplitude is the largest, but where the strain energy is maximum. The geometric discontinuity of the junction between the $\mathrm{ABH}$ profile and the uniform plateau of residual thickness, located at $x=155 \mathrm{~mm}$, seems to be an important source of mechanical power dissipation, as can be seen from the hot spot clearly visible on the early stage of the transient regime. 


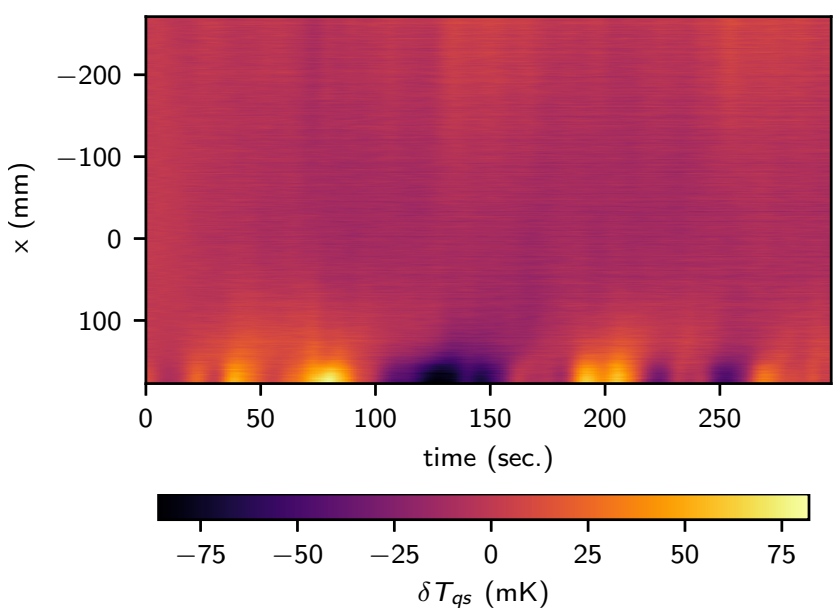

(a)

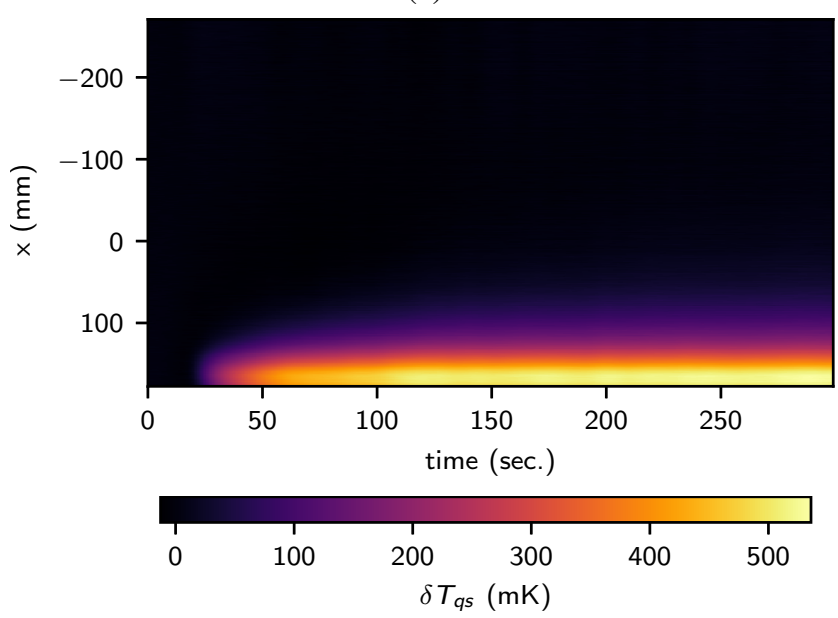

(b)

FIG. 9. Measured $\delta T_{q s}(x, t)$ for the (a) naked and (b) coated $\mathrm{ABH}$, with injected mechanical power described in Fig. 8

- the process of heat diffusion through the beam and of heat exchange with the surrounding fluid, result in a steady-state temperature field whose shape and amplitude depend on the frequency of excitation.

\section{CONCLUSION}

This paper concerns the experimental characterisation of damping mechanisms in vibrating structures, and especially in $\mathrm{ABH}$ endings. This issue is known to be tricky given the diversity and physical complexity of the dissipation mechanisms involved. The control and characterisation of damping is an important topic of research with several important stakes for the industry. In this context, the objective pursued here is to develop an imaging technique providing information on mechanical damping, based on the vibration and thermal maps of a vibrated structure.

The results reported in this paper focus on the thermogram measurement technique and its application to the case of an

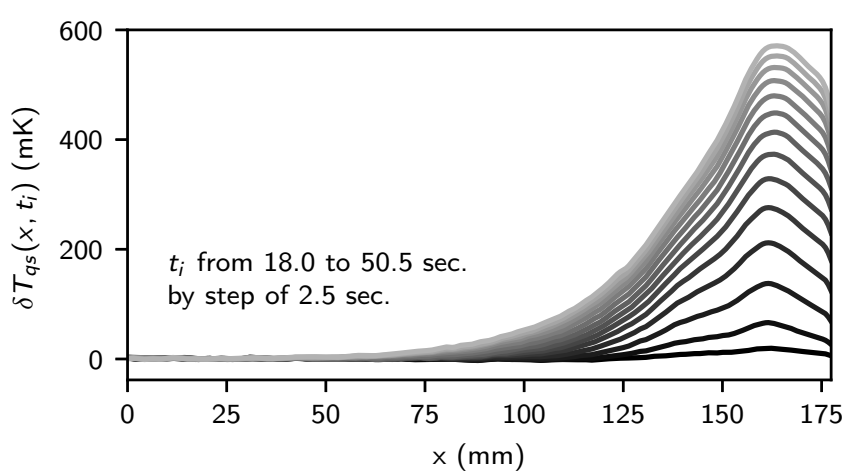

(a)

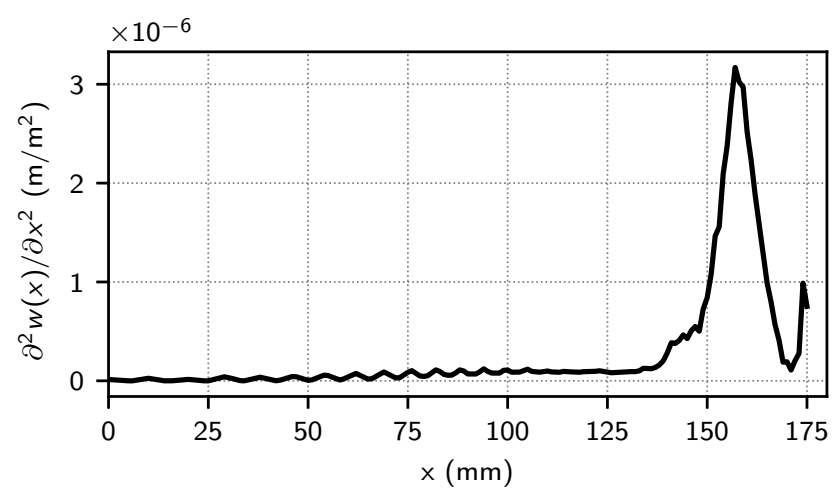

(b)

FIG. 10. (a) Measured $\delta T_{q s}\left(x, t_{i}\right)$ for $t_{i}$ ranging from (black) $18.0 \mathrm{sec}$. to (light grey) $50.5 \mathrm{sec}$. with time steps of $2.5 \mathrm{sec}$., and (b) estimated $\partial^{2} w(x) / \partial x^{2}$ at $276 \mathrm{~Hz}$.

ABH beam termination. The results are summarized below.

- An image processing method based on infra-red thermograms has been developed and enables carrying out the accurate detection of local heating induced by mechanical dissipation in a thin vibrating structure. The access to these new types of experimental data opens up potentially new approaches for both basic and applied research in the field of vibration.

- The evidence of a local temperature increase of a few hundreds of $\mathrm{mK}$, due to local damping in an $\mathrm{ABH}$ beam termination, is provided in the case of vibrations using a broadband noise signal.

- The analysis of the transient states, once the shaker is switched on, shows that the geometric discontinuity at the $\mathrm{ABH} /$ plateau junction is one of the important sources of heating.

The non-invasive techniques used in this paper provide useful information, which might help getting further insights on the complex processes of damping in vibrating structures. Indeed, damping usually occurs at junctions, and thermal imaging allows visualising this effect, and thus localising it. For example, this method could be applied to glazing with joints on borders or a panel with a partial visco-elastic coating or/and 


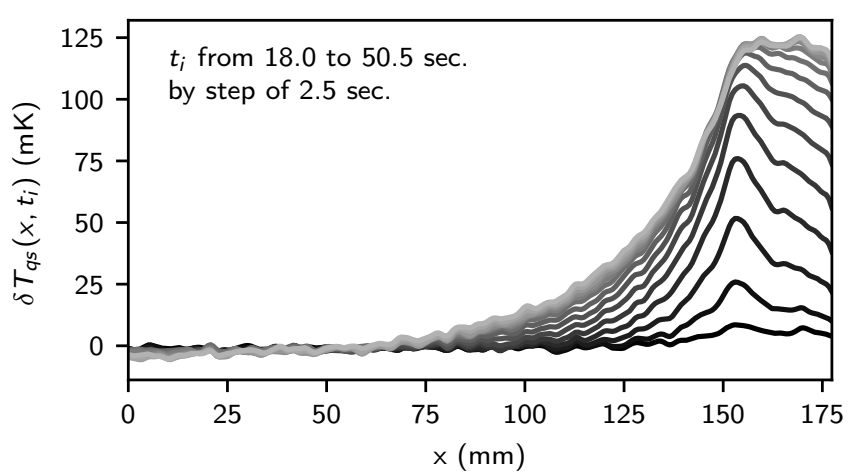

(a)

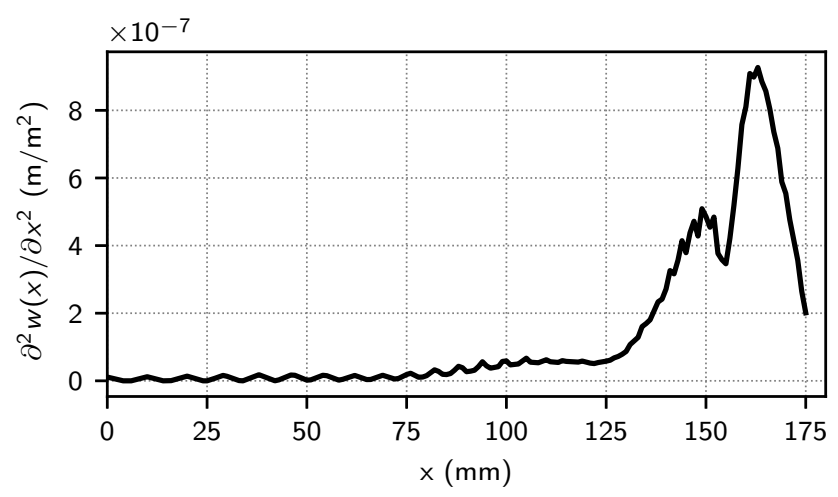

(b)

FIG. 11. (a) Measured $\delta T_{q s}\left(x, t_{i}\right)$ for $t_{i}$ ranging from (black) $18.0 \mathrm{sec}$. to (light grey) $50.5 \mathrm{sec}$. with time steps of $2.5 \mathrm{sec}$., and (b) estimated $\partial^{2} w(x) / \partial x^{2}$ at $933 \mathrm{~Hz}$.

installed on dampers. In the $\mathrm{ABH}$ case, data extracted from the thermograms and mechanical measurement may provide information to increase understanding on the $\mathrm{ABH}$ effect and may allow improving its characterisation. Works are currently in progress to develop a thermo-mechanical model enabling to reproduce the experimental observations reported in this paper.

\section{SUPPLEMENTARY MATERIALS}

Videos made with raw images obtained from the camera and with processed images corresponding to the data presented in Fig. 7, are available as supplementary materials, to present subjectively the quality of the signal and image processing applied.

\section{ACKNOWLEDGMENTS}

This work is funded by the French Research Agency (ANR) through the FullFields joint lab program (ANR-17- LCV20010). This work is also partly linked to the reasearch programme eTNAA funded by the ANR French agency (ANR17-CE08-0035) and dedicated to active control of damping in
ABH devices.

Authors are grateful to Julien Nicolas, from ENSIM Engineering School, who manufactured the ABH sample beam.

${ }^{1}$ M. A. Mironovv, "Propagation of a flexural wave in a plate whose thickness decreases smoothly to zero in a finite interval," Soviet Physics: Acoustics 34, 318-319 (1988).

${ }^{2} \mathrm{~V}$. V. Krylov, "New type of vibration dampers utilising the effect of acoustic 'black holes'," Acta Acustica united witth Acoustica 90, 830-837 (2004).

${ }^{3}$ V. Georgiev, J. Cuenca, F. Gautier, L. Simon, and V. Krylov, "Damping of structural vibrations in beams and elliptical plates using the acoustic black hole effect," Journal of Sound and Vibration 330, 2497-2508 (2011)

${ }^{4}$ V. Denis, A. Pelat, and F. Gautier, "Scattering effects induced by imperfections on an acoustic black hole placed at a structural waveguide termination," Journal of Sound and Vibration 362, 56- 71 (2016)

${ }^{5}$ L. Tang, L. Cheng, H. Ji, and J. Qiu, "Characterization of acoustic black hole effect using a one-dimensional fully-coupled and wavelet-decomposed semi-analytical model," Journal of Sound and Vibration 374, 172 - 184 (2016)

${ }^{6}$ J. Y. Lee and W. Jeon, "Exact solution of euler-bernoulli equation for acoustic black holes via generalized hypergeometric differential equation," Journal of Sound and Vibration 452, 191-204 (2019)

${ }^{7}$ V. Denis, F. Gautier, A. Pelat, and J. Poittevin, "Measurement and modelling of the reflection coefficient of an acoustic black hole termination," J. of Sound and Vibration 349, 67-79 (2015).

${ }^{8}$ A. Pelat, V. Denis, and F. Gautier, "Experimental and theoretical study of the reflection coefficient of a abh beam termination," in INTER-NOISE and NOISE-CON Congress and Conference Proceedings, Vol. 250 (Institute of Noise Control Engineering, 2015) pp. 5001-5009.

${ }^{9}$ O. Aklouche, A. Wang, A. Pelat, and F. Gautier, "Dispersion curves for bending waves in a meta-plate made with a periodic lattice of abh like scatterers," in INTER-NOISE and NOISE-CON Congress and Conference Proceedings, Vol. 255 (Institute of Noise Control Engineering, 2017) pp. 5226-5232.

${ }^{10}$ O. Aklouche, A. Pelat, S. Maugeais, and F. Gautier, "Scattering of flexural waves by a pit of quadratic profile inserted in an infinite thin plate," Journal of Sound and Vibration 375, 38-52 (2016)

${ }^{11}$ P. A. Feurtado and S. C. Conlon, "An experimental investigation of acoustic black hole dynamics at low, mid, and high frequencies," 138 (2016), $10.1115 / 1.4033894$

${ }^{12}$ L. Ma, S. Zhang, and L. Cheng, "A $2 d$ daubechies wavelet model on the vibration of rectangular plates containing strip indentations with a parabolic thickness profile,' Journal of Sound and Vibration 429, 130 - 146 (2018)

${ }^{13}$ J. Leng, F. Gautier, A. Pelat, R. Picó, J.-P. Groby, and V. Romero-García, "Limits of flexural wave absorption by open lossy resonators: reflection and transmission problems," New Journal of Physics 21, 053003 (2019)

${ }^{14}$ A. Pelat, F. Gautier, F. Semperlotti, and S. Conlon, "The acoustic black hole: a review of therory and applications," submitted to the Journal of Sound and Vibration (2019).

${ }^{15}$ A. Climente, D. Torrent, and J. Sánchez-Dehesa, "Gradient index lenses for flexural waves based on thickness variations," Applied Physics Letters 105 (2014), 10.1063/1.4893153

${ }^{16} \mathrm{~S}$. Foucaud, G. Michon, Y. Gourinat, A. Pelat, and F. Gautier, "Artificial cochlea and acoustic black hole travelling waves observation: Model and experimental results," Journal of Sound and Vibration 333, 3428 - 3439 (2014)

${ }^{17}$ T. Zhou, L. Tang, H. Ji, J. Qiu, and L. Cheng, "Dynamic and static properties of double-layered compound acoustic black hole structures," International Journal of Applied Mechanics 09, 1750074 (2017)

${ }^{18}$ T. Zhou and L. Cheng, "A resonant beam damper tailored with acoustic black hole features for broadband vibration reduction," Journal of Sound and Vibration 430, $174-184$ (2018)

${ }^{19}$ W. Huang, H. Zhang, D. J. Inman, J. Qiu, C. E. Cesnik, and H. Ji, "Low reflection effect by $3 \mathrm{~d}$ printed functionally graded acoustic black holes," Journal of Sound and Vibration 450, 96 - 108 (2019)

${ }^{20}$ M. Ouisse, D. Renault, P. Butaud, and E. Sadoulet-Reboul, "Damping control for improvement of acoustic black hole effect," Journal of Sound and Vibration 454, 63-72 (2019).

${ }^{21}$ F. Ablitzer, C. Pézerat, J.-M. Génevaux, and J. Bégué, "Identification of 
stiffness and damping properties of plates by using the local equation of motion," Journal of Sound and Vibration 333, 2454-2468 (2014).

${ }^{22}$ M. Mihalec, J. Javh, F. Cianetti, M. Moretti, G. Rossi, J. Slavič, and M. Boltežarn, "Damping heat coefficient - theoretical and experimental research on a vibrating beam," J. Sound Vib. 400, 13-21 (2017).

${ }^{23}$ G. Pitarresi and E. A. Patterson, "A review of the general theory of thermoelastic stress analysis," The Journal of Strain Analysis for Engineering Design 38, 405-417 (2003)

${ }^{24}$ G. Pitarresi, L. D'Acquisto, and A. M. Siddiolo, “Thermoelastic stress analysis by means of an infrared scanner and a two-dimensional fast fourier transform-based lock-in technique," The Journal of Strain Analysis for Engineering Design 43, 493-506 (2008)

${ }^{25} \mathrm{C}$. Antolis and N. Rajic, "Optical lock-in thermography for structural health monitoring - a study into infrared detector performance," Procedia Engineering 188, 471-478 (2017)

${ }^{26} \mathrm{~F}$. Li, J. Liu, H. Yang, Y. Lu, and L. Zhang, "Numerical simulation and experimental verification of heat build-up for rubber compounds," Polymer 101, 199-207 (2016)

${ }^{2 /}$ W. Luo, B. Yin, X. Hu, Z. Zhou, Y. Deng, and K. Song, "Modeling of the heat build-up of carbon black filled rubber,' Polymer Testing 69, 116-124
(2018)

${ }^{28} \mathrm{~N}$. Navaranjan and R. Jones, "Lock-in infrared thermography for the evaluation of the structural performance of corrugated paperboard structures," Composite Structures 92, 2525-2531 (2010)

${ }^{29}$ K. Akutagawa, S. Hamatani, and T. Nashi, "The new interpretation for the heat build-up phenomena of rubbery materials during deformation," Polymer 66, 201-209 (2015)

${ }^{30} \mathrm{~L}$. Tang and L. Cheng, "Enhanced acoustic black hole effect in beams with a modified thickness profile and extended platform," Journal of Sound and Vibration 391, 116-126 (2017).

${ }^{31}$ F. J. Fahy, "Measurement of acoustic intensity using the cross-spectral density of two microphone signals," The Journal of the Acoustical Society of America 62, 1057-1059 (1977)

${ }^{32}$ J. Y. Chung, "Cross-spectral method of measuring acoustic intensity without error caused by instrument phase mismatch," The Journal of the Acoustical Society of America 64, 1613-1616 (1978)

${ }^{35}$ A. V. Oppenheim and R. W. Schafer, Discrete-Time Signal Processing (Prentice-Hall, 1989) pp. 311-312.

${ }^{34}$ Z. Zembaty, P. Bobra, P. Bońkowski, S. Kokot, and J. Kuś, "Strain sensing of beams in flexural vibrations using rotation rate sensors," Sensors and Actuators A 269, 322-330 (2018). 